\title{
Más allá de la obediencia: reanálisis de la investigación de Milgram
}

\section{Beyond obedience: reanalysis of Milgram`s research}

\author{
Jesús M. Canto Ortiz y José L. Álvaro² \\ ${ }^{1}$ Departamento de Psicología Social, Antropología Social, Trabajo Social y Servicios Sociales de la Universidad de Málaga, España. \\ 2 Departamento de Psicología Social de la Universidad Complutense de Madrid, España.
}

Disponible online 30 de abril de 2015

\begin{abstract}
Los experimentos de Milgram sobre la obediencia a la autoridad son considerados una de las investigaciones más importantes de la psicología social. Su impacto ha abarcado tanto a la psicología social como a otras ramas de la psicología, incluidas otras ciencias sociales. En este trabajo se analiza la influencia recíproca de la investigación de Milgram y el concepto de la banalidad del mal de Arendt. Se critica el modelo teórico propuesto por Milgram y el concepto de la banalidad del mal de Arendt. No hay ninguna demostración empírica de la existencia del estado agéntico y de la relación entre éste y los niveles de obediencia obtenidos en los experimentos de Milgram. Además, obras recientes sobre el Holocausto no confirman que las acciones criminales de los líderes nazis se debieran a actos de obediencia debida. Son necesarios modelos teóricos como el que se basa en la perspectiva de la identidad social para explicar los datos obtenidos en el experimento de Milgram.
\end{abstract}

Palabras Clave: Autoridad; Holocausto; Milgram; Obediencia.

Milgram's obedience to authority study is considered one of the most important studies in social psychology. Its impact has gradually spread to social psychology, other branches of psychology, and other social sciences. This article analyses the reciprocal influence of Milgram's studies and Arendt's concept of the banality of evil. It also offers a critical analysis of Milgram's theoretical model (agentic state) and Arendt's concept of the banality of evil. It is suggested that there is no empirical evidence of the existence of an agentic state or a relationship between this state and the levels of obedience achieved in Milgram's experiments. Furthermore, recent studies on the Holocaust do not confirm that the criminal actions of the Nazi leaders were acts of due obedience. Theoretical models, such the one based on the perspective of social identity, are needed to explain the results obtained in Milgram's experiments.

Keywords: Authority; Holocaust; Milgram; Obedience.

Correspondencia: Jesús M. Canto Ortiz, Facultad de Psicología, Universidad de Málaga, Campus de Teatinos s/n Málaga, 290171. Teléfono: 65575 6213. Fax: 952131100. E-mail: jcanto@uma.es. E-mail del co-autor: José L. Álvaro: jlalvaro@hotmail.com 
Hace más de 50 años, el 7 de agosto de 1961, Stanley Milgram inició en la Universidad de Yale una serie de experimentos sobre la obediencia, constituyendo una de las investigaciones más influyentes de la psicología social (Álvaro y Garrido, 2003; Blass, 2004; Miller, 1986; Lunt, 2009). El Holocausto causado por los nazis durante el período de la Segunda Guerra Mundial marcó notablemente a las ciencias sociales, incluida la psicología social (Reicher, Haslam y Rath, 2008). El horror que provocó mostró que la sociedad industrial occidental no era más racional ni más civilizada que otras, sino que disponía de herramientas más sofisticadas para el asesinato y la barbarie. Milgram (1974) no quiso llevar a cabo un programa de investigación sobre la obediencia a la autoridad sin más, sino que intentó reproducir en sus experimentos distintos niveles de obediencia destructiva, tal y como tuvo lugar durante el gobierno de los nazis en Alemania.

Las investigaciones de Milgram quedan enmarcadas en la perspectiva psicosocial de la segunda mitad del siglo XX que resaltaba los efectos negativos de los grupos y ayudaron a consolidar el "sesgo de la conformidad", centrado en el control social y no en el cambio social (Moscovici, 1981). El énfasis en la investigación de los procesos de influencia social como la normalización (Sherif, 1936), la conformidad (Asch, 1956) y la obediencia (Milgram, 1974), en detrimento de la investigación de la influencia provocada por las minorías activas, la rebeldía o la resistencia (Moscovici, 1981), ha constituido un claro ejemplo de un sesgo de la investigación y de la teorización psicosocial, más preocupado por explicar el mantenimiento del status quo que el cambio y la transformación social (Canto, 1994; Haslam y Reicher, 2011; Parker, 2000).

Junto a las investigaciones de Milgram (1974), llevadas a cabo en la década de los 60 del siglo pasado, hubo otras importantes investigaciones que resaltaron la potencialidad de los grupos para provocar comportamientos agresivos, discriminatorios e irracionales. A principio de los años 50, Sherif y Sherif (1953) realizaron una investigación en un campamento de verano infantil y observaron que niños sin ningún rasgo psicopatológico fueron agresivos y crueles con antiguos amigos por el hecho de pertenecer a distintos grupos que competían entre sí. En esa misma década, Asch (1956) llegó a demostrar como las personas, estando en minoría, se ajustaban a los juicios de una fuente mayoritaria, aunque los juicios mayoritarios fuesen claramente erróneos. Junto con la aportación de Milgram (1974), la culminación de esta perspectiva que resaltaba los aspectos negativos de los grupos lo constituyó el experimento de la prisión de Stanford llevado a cabo por Zimbardo (Haney, Banks y Zimbardo, 1973). Los investigadores simularon una prisión en los sótanos de la universidad y asignaron a los estudiantes dos roles: el rol de "preso" o el rol de "guardián". El experimento fue diseñado para que durara dos semanas, pero fue suspendido al sexto día por la crueldad mostrada por varios "guardianes" contra los "presos". Zimbardo llegó a la conclusión que los individuos pierden su capacidad intelectual y su juicio cuando están en grupo y que hay una tendencia a abusar del poder en contextos grupales (para una visión crítica con este enfoque, véase Reicher y Haslam, 2006).

\section{La influencia del concepto de la banalidad del mal en la teoría de Milgram}

En buena medida, la enorme influencia de las investigaciones de Milgram (1974) se debió a que los resultados obtenidos fueron presentados como evidencia empírica del concepto de "la banalidad del mal" expuesto por Arendt (1963). Tras ser detenido en Argentina en 1960 por la policía secreta israelí, el 11 de abril de 1961, se inició en Jerusalén el juicio contra Eichmann, máximo responsable del plan para exterminar a los judíos por parte de los nazis. En la sala del juicio se encontraba la filósofa Hannah Arendt, que ya había escrito una importante obra sobre el autoritarismo (Arendt, 1951). Asistió a la primera fase del juicio como reportera de un periódico norteamericano y publicó en 1963 el libro titulado Eichmann in Jerusalem: A report on the banality of evil. La filósofa señaló que los psiquiatras asistentes al juicio describieron a Eichmann como un hombre obsesionado con matar. Sin embargo, Arendt lo que vio fue a un hombre gris, carente de ambiciones, sumamente obediente, un hombre que se sentía orgulloso del trabajo bien hecho, aunque su trabajo implicara acabar con la vida de millones de personas. Arendt no quiso decir con la expresión "la banalidad del mal" que los actos de exterminio cometidos por los nazis fueran banales, sino que los motivos que los sustentaban eran "banales", ya que estaban más centrados en realizar adecuadamente la tarea y agradar a sus superiores que en las consecuencias de sus actos. Esto explicaría que personas ordinarias pudieran cometer actos extremadamente crueles sin una motivación especial, sin una psicología de la personalidad distinta a la de la mayoría.

Arendt (1963) fue muy crítica con el comportamiento de los líderes judíos durante el Holocausto y ello le supuso ser objeto de fuertes críticas por parte de las autoridades de Israel. A pesar de tales críticas, su concepto de la banalidad del mal recibió apoyo por parte del modelo teórico adoptado por Milgram en sus investigaciones sobre obediencia. El propio Milgram se inspiró en el concepto de la banalidad del mal para explicar los niveles de obediencia obtenidos en sus investigaciones. Como afirmaron Reicher y Haslam (2012), el estudio histórico de Arendt sobre Eichmann y el Holocausto proporcionaron relevancia social a los estudios de Milgram y éstos proporcionaron credibilidad científica a las aportaciones de Arendt. Estos dos estudios, siendo de naturaleza muy diferente, se fusionaron para proporcionar un modelo sobre la naturaleza y maldad humanas, modelo que ha dominado el pensamiento científico y cultural durante más de medio siglo. Según Milgram él habría demostrado lo que Arendt observó en la sala donde se celebró el juicio contra el líder nazi: las personas ordinarias pueden infligir daños extraordinarios a otros seres humanos, por el simple hecho de prestar atención a las instrucciones de la autoridad y a la tarea y no a sus consecuencias. 


\section{Los hallazgos del Milgram}

En 1974 Milgram publicó su libro. También filmó sus experimentos y dirigió una película. Previamente (Milgram, 1963, 1965a, 1965b) había publicado diversos artículos donde exponía sus primeros hallazgos y el procedimiento empleado. Recientemente, Russell (2011, 2014) ha revisado documentalmente el proceso que llevó a Milgram a confeccionar sus experimentos. Influido por Asch, Milgram inició su carrera como investigador interesado en las diferencias nacionales en el fenómeno de la conformidad. Pero no estaba satisfecho con el procedimiento experimental utilizado por Asch (1956). No quería que "la prueba de la conformidad" versara sobre una tarea estimular (sobre la longitud de unas líneas) y se preguntó si los grupos tenían la capacidad de presionar a los individuos de tal forma que les obligaran a cometer actos dañinos contra personas inocentes, tal como tuvo lugar durante el Holocausto (Russell, 2011).

Milgram (1963) diseñó una situación experimental en la que cuarenta varones creyeron que estaban colaborando en una investigación sobre aprendizaje. Los participantes fueron seleccionados a través de anuncios de periódicos en la ciudad de New Haven y recibieron $4.5 \$$ por colaborar en el estudio. Cuando un sujeto experimental llegaba al laboratorio de la Universidad de Yale, se encontraba con un hombre de edad madura que declaraba que él también participaba en la experiencia. El experimentador, con bata blanca, les explicaba que la investigación trataba sobre la influencia del castigo en el aprendizaje. Tras un sorteo amañado, se le asignaba al sujeto experimental el rol de "profesor" y a la otra persona (cómplice del experimentador) el rol de "alumno". Una vez conectado a un dispositivo de electrodos, el experimentador informaba al profesor (sujeto experimental) que su tarea consistiría en leer pares de palabras al "alumno", verificar si era capaz de repetirlas e infligirle una descarga eléctrica por cada fallo. Para aplicar las descargas, se disponía de un generador con 30 conmutadores (de 15 en 15 voltios) que llegaba a los 450 voltios. Unos letreros colocados sobre los conmutadores llevaban inscripciones que iban desde "Descarga ligera" (de 15 a 60 voltios), "Descarga moderada" (de 75 a 120 voltios), "Descarga fuerte" (de 135 a 180), "Descarga muy fuerte" (de 195 a 240), "Descarga intensa" (de 255 a 300), "Descarga de extrema intensidad" (de 315 a 360), "Peligro: descarga intensísima (de 375 a 420)", "Descarga potente" (a los 375 voltios) y descarga extrema "XXX" (de 435 a 450). Durante el experimento, en el cual el castigo infligido era simulado, el "alumno" cometía numerosos errores y el "profesor" recibía instrucciones por parte del experimentador que debía ir aumentando progresivamente el nivel de descargas. El experimentador utilizaba cuatro instrucciones en función de la resistencia mostrada por el "profesor". Estas fueron: $1^{\text {a } ~ " P o r ~ f a v o r, ~}$ continúe", $2^{\text {a }}$ "El experimento requiere que prosiga", $3^{\text {a } ~ " E s ~}$ absolutamente esencial que prosiga" y $4^{\text {a }}$ "Usted no tiene otra opción, usted debe continuar". Además, había dos respuestas a dos posibles objeciones por parte del "profesor". Si éste pre- guntaba sobre el daño físico infligido al alumno, el experimentador contestaba: "Aunque las descargas pueden ser dolorosas, no producen daños permanentes en los tejidos, así que continúe". Y si el "profesor" objetaba que el "alumno" no quería continuar, el experimentador replicaba; "Tanto si el alumno quiere como si no, usted debe continuar hasta que el alumno haya aprendido todos los pares de palabras correctamente, así que continúe" (Milgram, 1974).

El "alumno" y el experimentador seguían un guión preestablecido. El "alumno" comenzaba a equivocarse y el experimentador daba instrucciones al "profesor" para que aplicara las descargas eléctricas pertinentes. Al llegar a 75 voltios, el "alumno" reflejaba dolor. A los 150 voltios, solicitaba dejar el experimento. A los 300 voltios, rehusaba seguir respondiendo y solicitaba abandonar el estudio. Y a los 330 voltios, dejaba de responder. En el experimento que constituyó la línea base (Milgram, 1963), obedecieron hasta los 450 voltios el 65\% de la muestra experimental (26 sujetos de 40). Muchos participantes reflejaron una fuerte tensión a lo largo del experimento, aún cuando Milgram diseñó el experimento con sumo cuidado para provocar que los sujetos fuesen obedientes hasta el final (Russell, 2011). Milgram (1974) realizó 18 variaciones de su diseño experimental (sin contar los estudios pilotos). Tras el análisis de los resultados obtenidos, se puede concluir que los niveles de obediencia en sus distintos estudios variaron del $0 \%$ al $90 \%$, en función de las diversas situaciones experimentales, tales como proximidad del "alumno", prestigio de la institución, número de experimentadores, desacuerdo entre los experimentadores, apoyo social para el "profesor", si el castigo lo demandaba el alumno, etc.

Milgram no disponía de un modelo teórico cuando inició sus estudios sobre la obediencia e, incluso, carecía de hipótesis antes de realizar el primer experimento (Russell, 2011). Fue al publicar su libro en 1974 cuando proporcionó una teoría, once años más tarde de su primer artículo sobre este tema. Milgram diferenció dos estados psicológicos para explicar por qué los individuos obedecían en sus experimentos: a) "el estado de autonomía”, en el cual las personas se sienten responsables de sus actos y utilizan su propia conciencia como guía de comportamiento y b) "el estado agéntico", en el cual las personas que se encuentran en este estado consideran que forman parte de una estructura jerárquica y sostienen que las autoridades son las responsables de sus actos y utilizan las órdenes de las autoridades como guía de acción correcta.

El concepto de estado agéntico implica que en las personas se da un cambio de autopercepción, una reorientación cognitiva que tiene lugar cuando ocupan un rol en una organización. Para Milgram (1974), el estado agéntico sería el responsable de la obediencia en las organizaciones, incluida la obediencia destructiva, tal como se podría observar en procesos históricos, tales como el Holocausto. Como afirmó Miller (1986), la "tesis de la normalidad" para explicar la obediencia de carácter destructivo no justificaría los crímenes producidos por este tipo de 
obediencia, sino que negaría la suposición de que las personas que comenten tales acciones destructivas y violentas fuesen a priori diferentes del resto de los individuos en términos de funcionamiento psicológico básico.

La explicación proporcionada por Milgram encajó plenamente en el concepto de la banalidad del mal de Arendt, al basarse en él. En definitiva, se podría sostener, siguiendo el planteamiento de Milgram, que Eichmann no fue un asesino por poseer un determinado tipo de psicopatología, sino que lo que fue, simplemente, por ser un burócrata eficiente que cayó en el estado agéntico al estar sometido a las órdenes de una autoridad. Para Milgram, someterse a las órdenes de una autoridad en un contexto jerárquico, al estar en el estado agéntico, sería un elemento constitutivo de la naturaleza del ser humano, una tendencia esencial para el buen funcionamiento de los grupos sociales.

\section{Repercusión de los estudios de Milgram (1974)}

El interés de Milgram por la obediencia destructiva se debió a factores académicos y personales (Russell, 2011). La influencia que ejerció sobre él Solomon Asch y, en menor medida su profesor Gordon Allport, hizo que se interesara por el estudio de la conformidad y, posteriormente por la obediencia (Blass, 2004). Razones de carácter personal también influyeron en su praxis investigadora. Milgram nació en 1933 en Nueva York, en el seno de una familia judía que emigró a EE.UU a principios del siglo XX. Como judío se preocupó por el sufrimiento de su pueblo durante la Segunda Guerra Mundial, causándole una gran impresión el Holocausto y las atrocidades cometidas por los nazis. Quería entender cómo fue posible que tantos oficiales de las SS hubieran matado a millones de personas de forma tan cruel cumpliendo órdenes de sus superiores.

Fueron muchas las razones por las que el trabajo de Milgram (1974) resultó tan relevante (Blass, 2004): En primer lugar, por el número elevado de sujetos que obedecieron en sus diseños experimentales. En segundo lugar, por llevar a cabo un programa de investigación tan amplio (con más de 18 variaciones experimentales). En tercer lugar, por las polémicas que suscitó. Y, en cuarto lugar, porque apoyó y dio pie a una concepción específica sobre el papel que podían desempeñar los factores situacionales en la conducta de los seres humanos. Milgram mostró en sus estudios la tendencia del ser humano a obedecer de forma acrítica a la autoridad, presentó un modelo explicativo y enseñó el enorme poder de la situación en la conducta humana, en detrimento de las disposiciones personales.

Milgram ejerció una notable influencia en la psicología de la personalidad (Benjamin y Simpson, 2009). En la década de los 50 del siglo pasado, esta rama de la psicología se centraba en la influencia de los rasgos de personalidad en la conducta humana. La preponderancia de las teorías internalistas de la personalidad se debía a la influencia de las teorías psicodinámicas como la desarrollada por Adorno, Frenkel-Brunswik, Levinson y Sanford (1950) sobre la personalidad autoritaria, con- cepto que fue utilizado para explicar la sumisión a la autoridad, la agresión a los exogrupos y el prejuicio. En la década de los 60 , los psicólogos de la personalidad viraron su foco de atención y se centraron principalmente en los factores situacionales para explicar la conducta humana. Este giro se debió, en parte, según Benjamin y Simpson (2009), a la influencia de los resultados de Milgram, que demostraban el poder de los factores situacionales, y al libro de Mischel (1968), donde se resaltaba la falta de evidencia empírica de la influencia de los rasgos de personalidad en el comportamiento humano.

La influencia de Milgram en la psicología social fue más notable (Benjamin y Simpson, 2009). En la década de los 70 la psicología social se centró prioritariamente en los efectos del poder de la situación. El trabajo de Milgram tuvo una clara influencia en el estudio del experimento de la prisión de Stanford (Haney et al., 1973). Que se resaltara la influencia de la situación quedó también reflejado cuando Wicker (1969) formuló un fuerte ataque al efecto de las actitudes en la conducta. La influencia de Milgram también se dejó notar en los procedimientos utilizados en las investigaciones psicosociales, modificándose el tipo de manipulaciones experimentales y aumentando el número de investigaciones llevadas a cabo en contextos naturales. Ello condujo al desarrollo de nuevos métodos no experimentales y de técnicas estadísticas más sofisticadas. Las críticas recibidas por Milgram sobre su procedimiento experimental (p.e. Baumrind, 1964; Masserman, 1968), cuestionándolo desde planteamientos éticos, provocaron la revisión del código ético en el campo de la investigación psicológica y psicosocial en Estados Unidos por la American Psychological Association. Para determinar la aceptación de los protocolos de investigación se tuvo especialmente en cuenta el consentimiento por parte de los participantes, la valoración de los riegos y beneficios que conlleva la participación y el tipo de "engaño" que se puede utilizar para camuflar o ocultar los verdaderos objetivos de la investigación (Benjamín y Simpson, 2009).

\section{Unos datos en busca de una teoría}

Los resultados obtenidos por Milgram (1974) fueron replicados en diversos países (Blass, 1999). Pero las críticas sobre las consideraciones éticas al paradigma experimental para investigar la obediencia produjeron la disminución y el posterior abandono de este tipo de investigaciones (Miller, 1986). Sin embargo, se han desarrollado diversas estrategias para vencer tales obstáculos (Reicher y Haslam, 2012): el uso de conductas menos dañinas (Meeus y Raaijmakers, 1986), el empleo de un paradigma de obediencia similar al empleado por Milgram pero reduciendo el nivel máximo de descargas eléctricas (Burger, 2009), el análisis de situaciones históricas de obediencia (Rochat y Modigliani, 1995) y el uso de simulaciones de realidad virtual del paradigma de Milgram (Slater et al., 2006), entre otras.

La mayor parte de las investigaciones sobre obediencia inspiradas en el paradigma experimental de Milgram han obtenido 
resultados similares. Investigaciones de carácter transcultural (Blass, 1991; Miller, 1986) e investigaciones más recientes desarrolladas en Estados Unidos (Burger, 2009) han concluido que el nivel de obediencia es similar en los distintos países y relativamente constante a lo largo del tiempo. Ello ha hecho que Blass (2004) afirmara que Milgram identificó uno de los universales de la conducta social: los seres humanos tendrían una tendencia a obedecer a la autoridad.

Sin embargo, Reicher y Haslam $(2011,2012)$ han cuestionado tanto las conclusiones elaboradas a partir de los datos obtenidos como la explicación teórica proporcionada por el propio Milgram (1974). Estos psicólogos sociales británicos resaltaron que se ha cometido un error en la interpretación de los resultados obtenidos sobre obediencia utilizando el procedimiento experimental de Milgram: se parte de la evidencia que las personas pueden obedecer a la mayoría de las instrucciones y órdenes y se acaba concluyendo que las personas no pueden dejar de obedecer a autoridades destructivas. El modelo explicativo propuesto por Milgram no ha resultado adecuado para explicar la obediencia (Darley, 1992; Ross, 1988). Milgram proporcionó una situación experimental diseñada con todo detalle para provocar obediencia (Russell, 2011), pero no proporcionó igualmente una explicación teórica satisfactoria. Para ciertos investigadores, más que un estudio sobre la obediencia, lo que constituyó fue una mera dramatización de la capacidad de las personas para ejercer violencia (Brannigan, 2004; Parker, 2000). La noción de estado agéntico sostiene que, ante una autoridad, las personas pierden de vista sus valores y normas y ceden la responsabilidad de sus actos a la autoridad, preocupándose tan sólo de seguir las instrucciones que proceden de la autoridad y no de las consecuencias de sus actos de obediencia. Pero como afirman Reicher y Haslam (2012), no hay evidencia empírica de que las personas entren en ese estado agéntico, como tampoco hay evidencia de que las variaciones en ese estado puedan explicar los distintos niveles de obediencia obtenidos en el laboratorio. Ni los resultados de Milgram (1974) ni el análisis histórico proporcionado por Arendt (1963), ampliamente rebatido por biografías más recientes sobre Eichmann, donde se demuestra que éste no fue un vulgar funcionario que cumplía órdenes de sus superiores, sino un nazi convencido, activo y dinámico en el ejercicio del poder (Cesarani, 2004), pueden ser utilizados para apoyar ni el concepto de la banalidad del mal ni la hipótesis del estado agéntico. Muchos de los participantes en los estudios de Milgram expresaron un elevado estrés y discutían tanto con el experimentador como con el "alumno", al tiempo que mostraban su oposición al realizar los actos que conllevaba obedecer. Tal como afirmó Cesarani (2004), la perspectiva que se derivó de Milgram y Arendt ha servido para impedir comprender adecuadamente el Holocausto y, por extensión, la maldad humana en general, estrangulando la investigación durante décadas.

Los niveles de obediencia obtenidos no dependen sólo del rol del experimentador (figura de autoridad al pertenecer a una universidad de reconocido prestigio), sino de las relaciones que se establecen entre los tres actores del paradigma de Milgram: el experimentador, el "alumno" y el sujeto experimental (Reicher y Haslam, 2011). Como afirmó Milgram (1965a), los participantes se hallaban en un dilema al tener que escuchar las peticiones del experimentador y las peticiones del "alumno". A medida que se hacía más presente la "voz" del "alumno", a medida que eran más audibles sus quejas, se hicieron más patentes las conductas de desobediencia. Packer (2008) ha demostrado que el momento más crítico para saber si un sujeto experimental va a ser obediente o no, es cuando se observaba lo que hacía cuando tenía que pulsar el conmutador de 150 voltios y, en menor medida, el conmutador de 315 voltios. A los 150 voltios, el "alumno" solicitaba abandonar el estudio y a los 315 voltios formalmente retiraba su consentimiento de seguir participando. Si obedecía, lo hacía hasta el final (450 voltios).

Milgram (1974) realizó variantes experimentales en las que el ambiente físico impactaba en la configuración de las relaciones sociales. Cuando manipuló la proximidad de la víctima, empleó en sus primeros artículos el término "formación de grupo incipiente" para explicar sus efectos en los niveles de obediencia (Milgram, 1965a). Según Reicher y Haslam (2011; 2012), cuando el sujeto experimental y el experimentador están juntos en la misma habitación y el "alumno" está en otra, es más probable que el participante se sitúe a sí mismo en la misma categoría social que el experimentador, mientras que resulta más improbable si el "alumno" también está con ellos. Al principio de cada estudio, los participantes se identificarían con el experimentador al ser el representante de la categoría "ciencia", por lo que estarían dispuestos a obedecer al compartir la misma identidad social (Turner, 1991). Reicher y Haslam (2011, 2012) propusieron a la teoría de la categorización del yo (Turner et al., 1987) como marco teórico para explicar los resultados obtenidos en el paradigma experimental de Milgram (1974). El concepto clave para comprender la influencia desde esta teoría es el concepto de influencia informativa referencial que versa sobre la influencia que se produce entre individuos y/o grupos que pertenecen a una misma categoría social, ya que, una vez se ha hecho saliente la categoría social pertinente, el individuo conoce las normas estereotipadas de la categoría y se las asigna, asumiendo una serie de conductas y opiniones que se derivan de aquélla (Turner, 1991). Una fuente de influencia (individuo o grupo) logra influir en la medida que es categorizada como endogrupo y, así, por esta coincidencia categorial, delimita las opiniones y conductas que son normativamente válidas. Desde esta óptica, según Reicher y Haslam (2011, 2012), sería conveniente investigar en el paradigma experimental de Milgram las diversas formas en las que el contexto impacta en las identidades sociales y el modo en el que éstas se ven determinadas por las demandas del experimentador y del "alumno". Además, se debería analizar los elementos discursivos utilizados por los participantes para desafiar los requerimientos del experimentador y desobedecer a las órdenes 
de éste. Milgram (1965a) ya señaló que una de las razones por la que los participantes seguían obedeciendo se debía a que carecían de recursos retóricos para desafiar la legitimidad de las demandas del experimentador (véase Haslam, Reicher y Birney, 2014; Reicher, Haslam y Smith, 2012).

Así, pues, como sostienen Reicher, Haslam y Smith (2012), la obediencia a la autoridad (incluida la obtenida con el paradigma experimental de Milgram) debe ser explicada por los niveles de identificación social activados en la situación grupal generadora de obediencia. En las variaciones del paradigma de Milgram habrían condiciones que fomentarían la identificación con el experimentador (y, por lo tanto, con la comunidad científica), mientras otras fomentarían la identificación con el "alumno" (y, por extensión, con la comunidad en general). La complacencia de los participantes en llevar a cabo una conducta destructiva no se deriva de un simple acto de pérdida de conciencia moral, sino de una identificación activa con el experimentador y la misión científica que realiza y con la cual él se compromete.

\section{Conclusiones}

No cabe duda que el programa de investigación diseñado por Milgram (1974) para estudiar la obediencia debe ser considerado como una de las aportaciones más sobresalientes de la psicología social. Su impacto fue muy importante al resaltar "el poder de la situación", lo que influyó en la disminución del valor explicativo atribuido a las variables de personalidad, primando la importancia del contexto social como factor determinante. El trabajo de Milgram coincidía plenamente con el Zeitgeist de la época, imbuido por las tesis conductistas en psicología (Mischel, 1968).

Junto con las investigaciones de Asch (1956) sobre conformidad, la investigación de Milgram ha constituido un claro exponente del sesgo de la psicología social que enfatizaba los procesos de influencia social responsables del control social (Moscovici, 1981): los individuos son débiles blancos de influencia ante las mayorías y ante las autoridades, sucumbiendo a la presión del grupo. El trabajo de Milgram no sólo conllevó una visión de la influencia social propensa a generar uniformidad y control social, sino que contribuyó a desarrollar una visión negativa, psicopatologizada, del grupo, que se había iniciado con la psicología de las masas (LeBon, 1895) y que continuó a lo largo del siglo XX.

Milgram (1974) presentó una propuesta sobre la importancia de los factores contextuales en la obediencia y el origen de la maldad humana, basada en el concepto de la banalidad del mal de Arendt (1963) y respaldada por la investigación de Zimbardo (Haney et al., 1973). La propia explicación de Milgram sobre la obediencia destructiva ("los participantes pasaron de un estado de autonomía a un estado agéntico") y la propia explicación de Zimbardo ("fue el uniforme de los policías lo que le hicieron comportarse de forma violenta hacia los presos, como un claro ejemplo de desindividuación") conllevaron el peligro de justificar la tiranía sin explicarla (Reicher y Haslam, 2012). No se debe exagerar la significación de los estudios de Milgram para una adecuada comprensión de la obediencia destructiva de los nazis para generar el Holocausto. No se puede explicar el grado de obediencia alcanzada en la Alemania nazi como un caso de "obediencia debida" donde los nazis obedecieron a una autoridad que les solicitaba actos de maldad de forma secuencial hasta llegar al exterminio de millones de personas. Los nazis obedecieron a sus líderes porque se identificaban con ellos y aceptaban la ideología nazi y estimaban que estaban realizando lo correcto cuando exterminaban a otras personas (incluidas no sólo las personas de exogrupos, sino también personas del endogrupo, como a alemanes de ideologías de izquierda, alemanes con minusvalías, homosexuales, etc.). La falta de continuidad en el tiempo de los actos de obediencia en los experimentos de Milgram, lo inesperado del tipo de órdenes emitidas por el experimentador (figura de autoridad) y la ambigüedad de la situación (Orne y Holland, 1968) hacen que no sean comparables los actos genocidas que han tenido lugar en la Historia y la investigación de Milgram.

Los experimentos de Milgram fueron diseñados cuidadosamente para provocar obediencia (Russell, 2011, 2014). Fueron realizados de tal forma que una autoridad legítima (un experimentador representante de la comunidad científica) iría dando órdenes cada vez más ilegítimas, de naturaleza más destructiva. Ha sido ampliamente constatado que la obediencia se reducía drásticamente cuando el experimentador proporcionaba la orden número cuatro ("Usted no tiene otra opción, usted debe continuar"), orden que expresamente se alejaba de las exigencias de la lógica científica y el experimentador quería imponerse más taxativamente (Reicher y Haslam, 2011).

Aunque se ha prestado más atención a los niveles de obediencia, la investigación de Milgram debe ser contemplada también como un ejemplo de que la desobediencia es posible.

En los experimentos de Milgram (1974) se introdujeron importantes diferencias situacionales e individuales que incidieron en los niveles de obediencia y desobediencia (Burger, 2009). La desobediencia es una respuesta a la autoridad como lo es la obediencia. A lo largo de la Historia se observa la presencia de múltiples individuos y grupos que se han resistido a la autoridad, que han desobedecido y se han organizado para combatir a la autoridad y al orden social con el que estaban en desacuerdo. Y muchas personas han puesto su vida en peligro en tales acciones. Cualquier modelo teórico que intente explicar la obediencia y la desobediencia deberá tener en cuenta el contexto grupal en el que tenga lugar la relación entre la figura de autoridad y los miembros del grupo, las estrategias utilizadas para conseguir ser obedecida y el grado de identificación de los miembros del grupo con su grupo y con la autoridad (Reicher y Haslam, 2011, 2012). Además, se deberá prestar atención al grado de interiorización de la obediencia, si es asumida plenamente o simulada, a las distintas respuestas de desobediencia (pasiva o activa) y a las consecuencias de la misma. Tanto 
la obediencia como la desobediencia ante las órdenes de una autoridad (legítima o no) son opciones, porque siempre es posible la resistencia en los procesos de influencia. La Historia lo demuestra, los experimentos de Milgram también.

\section{Limitaciones del estudio}

La propuesta de Haslam y Reicher está propiciando el desarrollo de un programa de investigación que ha presentado sus primeros trabajos en el análisis de situaciones de obediencia desde la perspectiva de la identidad social (Haslam, Reicher y Birney, 2014; Reicher, Haslam y Smith, 2012). Sin embargo, la revisión crítica de los resultados y del modelo teórico elaborado por Milgram abarca a más líneas de investigación que las llevadas a cabo por Haslam y Reicher y a más disciplinas que a la propia psicología social (Reicher, Haslam y Miller, 2014). La disponibilidad de los archivos de las investigaciones llevadas a cabo por Milgram (Russell, 2011, 2014) ha posibilitado el estudio de la interacción entre los participantes y el experimentador en algunos de los experimentos de Milgram (Gibson, 2014), así como el análisis de ciertos experimentos no publicados por este psicólogo social (Rochat y Blass, 2014), en los que se presta atención a los niveles de desobediencia y a los factores que la originan.

La realización de investigaciones sobre obediencia en las que se emplean niveles inferiores de descargas eléctricas (Burger, 2009) está siendo importante para seguir analizando los factores determinantes de los procesos de obediencia y desobediencia desde modelos teóricos más próximos a la psicología social que el elaborado por el propio Milgram.

\section{Referencias}

1. Adorno, T., Frenkel-Brunswik, E., Levinson, D. y Sanford, N. (1950). The authoritarian personality. Nueva York: Harper.

2. Álvaro, J. L. y Garrido, A. (2003). Psicología social. Perspectivas psicológicas y sociológicas. Madrid: McGraw Hill.

3. Arendt, H. (1951). Origins of totalitarianism. San Diego, CA: Harvest.

4. Arendt, H. (1963). Eichmann in Jerusalem: A report on the banality of evil. Nueva York: Penguin.

5. Asch, S. (1956). Studies of independence and conformity: A minority of one against a unanimous majority. Psychological Monographs: General and Applied, 70, 1-70. http:// dx.doi.org/10.1037/h0093718

6. Baumrind, D. (1964). Some thoughts on the ethics of research: After reading Milgram's "Behavioral Study of Obedience". American Psychologist, 19, 421-423. http:// dx.doi.org/10.1037/h0040128

7. Benjamin, L. y Simpson, J. (2009). The power of situation. The impact of Milgram's obedience studies on personality and social psychology. American Psychologist, 64, 12-19. http://dx.doi.org/10.1037/a0014077
8. Blass, T. (1991). Understanding behaviour in the Milgram obedience experiment: The rol of personality, situations, and their interactions. Journal of Personality and Social Psychology, 60, 398-413. http://dx.doi.org/10.1037/00223514.60.3.398

9. Blass, T. (1999). The Milgram paradigm after 35 years: Some things we now know about obedience to authority. Journal of Applied Social Psychology, 29, 955-978. http:// dx.doi.org/10.1111/j.1559-1816.1999.tb00134.x

10. Blass, T. (2004). The man who shocked the world: The life and legacy of Stanley Milgram. Nueva York: Basic Books.

11. Brannigan, A. (2004). The rise and the fall of social psychology: The use and misuse of the experimental method. New Jersey: Aldine Transaction.

12. Burger, J. (2009). Replicating Milgram. Would people still obey today? American Psychologist, 64, 1-11. http://dx.doi. org/10.1037/a0010932

13. Canto, J. M. (1994). Psicología social e influencia. Málaga: Aljibe.

14. Cesarani, D. (2004). Eichmann: His life and crimes. Londres: Heinemann.

15. Darley, J. (1992). Social organization for the production of evil. Psychology Inquiry, 3, 199-218. http://dx.doi. org/10.1207/s15327965pli0302_28

16. Gibson, S. (2014). Discourse, defiance, and rationality: "Knowledge work" in the "obedience" experiments. Journal of Social Issues, 70, 424-438. http://dx.doi.org/10.1111/ josi.12069

17. Haney, C., Banks, C. y Zimbardo, P. (1973). A study of prisoners and guards in a simulated prison. En E. Aronson (Ed.), Readings about the social animal (pp. 52-67). San Francisco, CA: Freeman.

18. Haslam, S. y Reicher, S. (2011). Beyond conformity. Revisiting classic studies and exploring the dynamics of resistance. En J. Jetten y M. Hornsey. Rebels in groups: dissent, deviance, difference and defiance (pp. 324-344). Londres: Wiley-Blackwell.

19. Haslam, S., Reicher, S. y Birney, M. (2014). Nothing by mere authority: evidence that in an experimental analogue of the Milgram paradigm participants are motivated not by orders but by appeals to science. Journal of Social Issues, 70, 473-488. http://dx.doi.org/10.1111/josi.12072

20. Le Bon, G. (1895). Psychologie des foules. Félix Alcan: París.

21. Lunt, P. (2009). Stanley Milgram. Understanding obedience and its implications. Nueva York: Palgrave MacMillan

22. Masserman, J. (1968). Debatable conclusions. International Journal of Psychiatry, 6, 261-281.

23. Meeus, W. y Raaijmakers, Q. (1986). Administrative obedience: Carrying out orders to use psychological-administrative violence. European Journal of Social Psychology, 16, 311-324. http://dx.doi.org/10.1002/ejsp.2420160402

24. Milgram, S. (1963). Behavioral study of obedience. Jour- 
nal of Abnormal and Social Psychology, 67, 371-378. http://dx.doi.org/10.1037/h0040525

25. Milgram, S. (1965a). Some conditions of obedience and disobedience to authority. Humans Relations, 18, 57-76. http://dx.doi.org/10.1177/001872676501800105

26. Milgram, S. (1965b). Liberating effects of group pressure. Journal of Personality and Social Psychology, 1, 127-134. http://dx.doi.org/10.1037/h0021650

27. Milgram, S. (1974). Obedience to Authority: An experimental view. Nueva York: Harper and Row.

28. Miller, A. (1986). The obedience experiments: A case study of controversy in social science. Nueva York: Praeger.

29. Mischel, W. (1968). Personality and assessment. Nueva York: Wiley.

30. Moscovici, S. (1981). Psicología de las minorías activas. Madrid: Morata.

31. Orne, M. y Holland, C. (1968). On the ecological validity of laboratory deceptions. International Journal of Psychiatry, 6, 282-293.

32. Packer, D. J. (2008). Identifying systematic disobedience in Milgram's obedience experiments: A meta-analytic review. Perspectives on Psychological Science, 3, 301-304. http:// dx.doi.org/10.1111/j.1745-6924.2008.00080.x

33. Parker, I. (2000). Obedience. Granta, 71, 99-124.

34. Reicher, S. y Haslam, S. (2006). Rethinking the psychology of tyranny: The BBC prison study. British Journal of Social Psychology, 45, 1-40. http://dx.doi. org/10.1348/014466605X48998

35. Reicher, S. y Haslam, S. (2011). After shock? Towards a social identity explanation of the Milgram 'obedience' studies. British Journal of Social Psychology, 50, 163-169. http://dx.doi.org/10.1111/j.2044-8309.2010.02015.x

36. Reicher, S. y Haslam, S. (2012). Obedience. Revisiting Milgram's shock experiments. En J. Smith y S. Haslam (Eds.), Social Psychology. Revisiting the classic studies (pp. 106-125). Londres: Sage.

37. Reicher, S., Haslam, S. y Miller, A. G. (2014). What makes a person a pertetrator? The intellectual, moral, and methodological arguments for revisiting Milgram's research on the influence of authority. Journal of Social Issues, 70, 393409. http://dx.doi.org/10.1111/josi.12067

38. Reicher, S., Haslam, S. y Rath, R. (2008). Making a virtue of evil: A five-step social identity model of the development of collective hate. Social and Personality Psychology Compass, 2/3, 1313-1344. http://dx.doi.org/10.1111/j.17519004.2008.00113.x
39. Reicher, S., Haslam, S. y Smith, J. (2012). Working towards the experimenter: reconceptualizing obedience within the Milgram paradigm as identification-based followership. Perspectives on Psychology Science, 7, 315-324. http:// dx.doi.org/10.1177/1745691612448482

40. Rochat, F. y Midigliani, A. (1995). The ordinary quality of resistance: From Milgram's laboratory to the village of Le Chambon. Journal of Social Issues, 51, 195-210. http:// dx.doi.org/10.1111/j.1540-4560.1995.tb01341.x

41. Rochat, F. y Blass, T. (2014). Milgram's unpublished obedience variation and its historical relevance. Journal of Social Issues, 70, 456-472. http://dx.doi.org/10.1111/ josi.12071

42. Ross, L. D. (1988). Situationist perspectives on the obedience experiments. Contemporary Psychology, 33, 101104.

43. Russell, N. J. (2011). Milgram's obedience to authority experiments: Origins and early evolution. British Journal of Social Psychology, 50, 140-162. http://dx.doi. org/10.1348/014466610X492205

44. Russell, N. J. (2014). The emergence of Milgram`s bureaucratic machine. Journal of Social Issues, 70, 409-423. http://dx.doi.org/10.1111/josi.12068

45. Sherif, M. (1936). The psychology of social norms. Nueva York: Harper \& Row.

46. Sherif, M. y Sherif, C. (1953). Groups in harmony and tension. Nueva York: Harper \& Row.

47. Slater, M., Antley, A., Davison, A., Swapp, D., Guger, C., Barker, C., Pistrang, N. y Sanchez-Vives, M. V. (2006). A virtual reprise of the Stanley Milgram obedience experiments. PloS ONE, 1: e39. http://dx.doi.org/10.1371/journal.pone.0000039

48. Turner, J. C. (1991). Social influence. Buckingham: Open University Press.

49. Turner, J.C., Hogg, M. A., Oakes, P. J., Reicher, S. y Wetherell, M. S. (1987). Rediscovering the social group. A selfcategorization theory. Oxford: Basil Blackwell.

50. Wicker, A. (1969). Attitudes versus actions: The relationship between verbal and overt behavioural response in attitude objects. Journal of Social Issues, 25, 41-78. http:// dx.doi.org/10.1111/j.1540-4560.1969.tb00619.x

Fecha de recepción: 18 de julio, 2014

Fecha de recepción de la versión modificada: 29 de noviembre, 2014

Fecha de aceptación: 7 de enero, 2015 